\title{
Experience of augmenting critical care capacity in Daegu during COVID-19 incident in South Korea
}

\author{
Je Hyeong Kim', Suk-Kyung Hong ${ }^{2}$, Younghwan Kim³ ${ }^{3}$ Ho Geol Ryu ${ }^{4}$, Chi-Min Park ${ }^{5}$, Young Seok Lee ${ }^{6}$, \\ Sung Jin Hong ${ }^{7}$ \\ ${ }^{1}$ Department of Critical Care Medicine, Korea University Ansan Hospital, Korea University College of Medicine, Ansan; ${ }^{2}$ Division of Acute Care Surgery, \\ Department of Surgery, Asan Medical Center, University of Ulsan College of Medicine, Seoul; ${ }^{3}$ Department of Surgery, National Medical Center, Seoul; \\ ${ }^{4}$ Department of Anesthesiology and Pain Medicine, Seoul National University Hospital, Seoul National University College of Medicine, Seoul, ${ }^{5}$ Department of \\ Critical Care Medicine, Samsung Medical Center, Sungkyunkwan University School of Medicine, Seoul; ${ }^{6}$ Division of Pulmonology and Critical Care Medicine, \\ Department of Internal Medicine, Korea University Guro Hospital, Korea University College of Medicine, Seoul, 7 Department of Anesthesiology and Pain \\ Medicine, Yeouido St. Mary's Hospital, College of Medicine, The Catholic University of Korea, Seoul, Korea
}

\section{BACKGROUND}

South Korea confirmed its first case of Coronavirus Disease-19 (COVID-19) on January 20, 2020. Starting from the outbreak of confirmed cases at a religious group in Daegu on February 18, 2020, the accumulated number of confirmed cases spiked from three digits to four between February 26 and April 3, 2020. The death rate continued to rise since the first reported death on February 20, 2020. The number of newly confirmed case per day has declined since hitting a record high of 909 on February 29, dropping to less than 10 since May 4. As of May 15,2020 , the overall death rate of COVID-19 in South Korea is $2.36 \%$, with $74.8 \%$ of the total confirmed cases and $89.6 \%$ of deaths occurring in Daegu and Gyeongsangbuk-do [1]. The regional bias of the entire confirmed and deceased was difficult to handle with medical resources in Daegu and Gyeongsangbuk-do, and it is believed that there would have been greater difficulties in intensive care, especially for critically ill patients.

The Korean Society of Critical Care Medicine (KSCCM) has formed a task force team (TFT) since March 2 and began discussing measures for critical care, following the increase in the number of patients with COVID-19. According to a report of China [2], one of the main risk factors of COVID-19-related deaths is old age, and the median time from symptoms onset to intensive care unit (ICU) admission was 10.5 days. In South Korea, 19.1\% of all confirmed cases were patients aged 60 or older on March 1, and considering the situation in which the daily number of newly confirmed cases showed the highest on February 29, the number of serious patients could be predicted to increase sharply around March 10. The KSCCM judged that a rapid increase in the number of critically ill patients in Daegu and Gyeongsangbuk-do would be difficult for local medical institutions to care for them. To establish a system for transferring and treat critically ill patients to other regions, KSCCM started to identify available ICU beds for COVID-19 critically ill patients and contact and discuss with the relevant government authorities [3].

\section{Brief Communication}

Received: May 19, 2020

Revised: May 26, 2020

Accepted: May 29, 2020

Corresponding author

Sung Jin Hong

Department of Anesthesiology and

Pain Medicine, Yeouido St. Mary's

Hospital, College of Medicine, The

Catholic University of Korea,

10 63(yuksam)-ro, Yeongdeungpo-

gu, Seoul 07345, Korea

Tel: +82-2-3779-1097

E-mail: hongs@catholic.ac.kr

Copyright (๑) 2020 The Korean Society of Critical Care Medicine

This is an Open Access article distributed under the terms of Creative Attributions Non-Commercial License (https:/I creativecommons.org/li-censes/by-nc/4.0/) which permits unrestricted noncommercial use, distribution, and reproduction in any medium, provided the original work is properly cited. 


\section{DAEGU DONGSAN HOSPITAL}

Keimyung University Daegu Dongsan Hospital is located in the center of Daegu Metropolitan City. Prior to April 2019, the hospital was operated as tertiary general hospital with a total of 1,000 beds and 50 ICU beds, but faced COVID-19 while it was being operated as a secondary general hospital due to the construction and relocation of the main hospital of Keimyung University Medical Center. On February 21, 2020, following the rapid increase in the number of confirmed cases in Dae$\mathrm{gu}$, the hospital was designated as a regional center hospital dedicated to treating COVID-19 cases with cohort isolation. At first, mild patients were mainly hospitalized, but clinically deteriorating patients began to occur among patients with advanced age and underlying diseases. Unfortunately, they could not be transferred to other hospitals because most of Daegu's other tertiary general hospitals' ICUs were fully occupied. Furthermore, because of the severity of the patients, the risk of long-distance transport to other regions was very high, and an appropriate transfer system was not established. Medical personnel in Daegu were not able to be recruited because they were dedicated to the treatment of serious patients who occurred or visited each hospital. Therefore, officials at Daegu Dongsan Hospital actively sought to expand beds, facilities, equipment and medical personnel for intensive care in order to treat serious patients occurring in the hospital on their own [4].

Because the situation of medical institutions in Daegu was very poor, and proper measures of government-related institutions could not be expected, Daegu Dongsan Hospital began discussions with Global Care, a healthcare non-governmental organization (NGO), to expand its intensive care capabilities. Global Care has provided funds for the introduction of essential equipment in cooperation with the Daegu Community Chest of Korea, while contacting various medical-related organizations to recruit intensive care medical personnel. However, as there were no organizations or medical centers that could recruit medical personnel, Global Care examined the possibility of supporting medical personnel to KSCCM, a multidisciplinary academic organization related to intensive care.

\section{COLLABORATION BETWEEN KSCCM AND DAEGU DONGSAN HOSPITAL FOR COVID-19 CRITICALLY ILL PATIENTS}

On March 6, 2020, considering the urgency of the issue, the KSCCM discussed the proposal of Global Care at the TFT meet- ing and decided to recruit volunteers, and organize dispatched medical team as quickly as possible. After then, for about three days, the KSCCM notified its members, sent requests to hospitals and relevant government authorities for formal process, and held discussions with Daegu Dongsan Hospital and Global Care [4]. As a result, on March 10, the first critical care team consisting of six intensivists and 11 intensive care nurses was dispatched to Daegu Dongsan Hospital.

After arriving at Daegu Dongsan Hospital, the medical team held a meeting with hospital officials to find out the current status of intensive care such as the number of critically ill patients and medical staffs, the size of ICUs, beds, facilities, and equipment. In addition, detailed plans were established for adding more beds as well as expanding and implementing facilities and equipment. Before participating in the medical treatment, the dispatched medical personnel received education for wearing personal protective equipment (PPE) to prevent infection and related education about the computer system for identifying various test results and prescribing medications. After the meeting and training, the medical team wore PPE and entered the ICU of a hospital isolated by cohorts, checked the status, and began treatment on the day of arrival.

The number of ICU beds had increased from three to seven. The number of serious patients is expected to increase rapidly in the near future, so it has decided to expand to 10 beds in a short period of time and 20 beds in 2 weeks. In order to expand the beds, the existing ICUs were reorganized to establish a minimum separation space for preventing infection of the medical staffs, a computer system, and the medical gas facilities such as oxygen. As medical equipment for operating expanded beds, specific numbers of equipment including mechanical ventilators, high-flow nasal canula, and continuous renal replacement therapy were decided and introduced. The extracorporeal membrane oxide (ECMO) equipment has no domestic inventory available for purchase, so it has borrowed equipment not used in other hospitals in Daegu.

When KSCCM critical care team arrived at the hospital, one intensivist and a few nurses were in charge of intensive care treatment. Local and dispatched intensivists, military doctors, and public health doctors who volunteered to work in ICUs set up a 24-hour work schedule for intensive care a day. Critical care nurses also began their three-shift work with local nurses and nurses who volunteered on different routes. Critical care nurses were not allowed to work more than 2 hours wearing PPE, so they worked alternately every 2 hours per work schedule. In order to ensure continued expansion of the volunteer medical staffs, the KSCCM recruited medical per- 
sonnel through repeated notices on all members, and as a result 21 intensivists and 11 critical care nurses volunteered for medical collaboration during the 6 weeks leading up to April 19.

A wide range of measures must be taken to provide necessary critical care because doctors and nurses have diverse areas of expertise and experience. The measures included setting triage standards to select patients who need to be monitored and treated in an ICU and address patients whose medical condition have improved enough to be transferred to a general ward. Intubation and tapering of supplemental oxygen therapy standards and goals for ECMO patient management were also set. The vasopressor, inotropic agent, analgesic, and sedative prescription methods also had to be standardized because each hospital had different protocols. In the absence of an effective treatment for COVID-19, based on reports and treatment guidelines of drugs that have been treated in China and other countries, the decision was made by discussing possible effective treatment drugs.

In the care of patients with infectious diseases, such as COVID-19, measures to prevent infection by the medical staffs are essential. Infection prevention is more important in situations where patients with high emissions of viruses need to be treated or treated in proximity for a long time. It is advantageous for intensive care personnel working in contaminated atmospheric conditions to wear a powered air-purifying respirator (PAPR), a type of PPE. Disposable hoods are needed to wear PAPRs, and in this COVID-19 incident, the medical personnel had to sterilize hoods themselves and wear them for several times or more than 1 week due to a lack of stockpiles. However, it is highly likely that the medical personnel's direct disinfection is not sufficient when the hood is worn, so additional personnel dedicated to disinfection and drying have been requested and deployed. A total of 33 patients were treated over 6 weeks in an improved and settled collaboration care system through these processes. Fourteen of them applied mechanical ventilation and three received ECMO treatment.

\section{PROPOSALS}

This medical collaboration is an unprecedented case in which local hospitals, professional medical staffs from an academic society, national medical institutions, private medical institutions, a healthcare NGO, and the Community Chest of Korea established a cooperative system to augment critical care capacity to treat critically ill patients in the face of disaster caused by a new infectious disease.

The COVID-19 crisis is a critical situation currently under- way in South Korea and worldwide, with the possibility of sporadic outbreaks of severe cases, specific regional concentrations, and nationwide mass outbreaks. Therefore, stepwise and comprehensive measures should be devised systematically.

This critical care collaboration was designed to effectively respond to the concentration of critically ill patients in a specific region by augmenting the critical care capacity of local hospitals under the conditions of the lack of beds in existing ICUs and the nonexistent patient transfer system. Situations like these should see the prioritization of securing facilities large enough to install additional hospital beds. In Italy, Spain, United Kingdom, and United States, where the number of patients with COVID-19 increased dramatically to an unbearable level of local medical capacity, gyms or convention centers were used as facilities to expand beds. In the case of Daegu, fortunately, the existing ICUs of Daegu Dongsan Hospital were in an advantageous position to expand beds for intensive care in a timely manner.

In the event of a rapid increase in the number of critically ill patients in a particular area, intensive care personnel in the region will be burdened with excessive medical care, and even if beds are expanded, they will not be able to perform or sustain care of patients. In this situation, medical personnel should be recruited from other regions. In this medical collaboration, the academic society went beyond its own role and actively recruited and dispatched volunteer medical personnel using its own human network, supporting local medical personnel in difficult situations and establishing a cooperative system. However, a way to rely on volunteers cannot guarantee the stable continuity of the medical workforce. Therefore, relevant government authorities need to play a central role in policymaking and developing plans for the recruitment and management of medical professionals required for effective and sustainable treatment of critically ill patients.

For medical personnel to properly treat critically ill patients in expanded beds, essential equipment for the treatment of critical patients must be secured. In this collaboration, the healthcare NGO and the Community Chest of Korea raised funds to introduce insufficient equipment in a timely manner, and some institutions and companies donated their own equipment. However, in cases where there is no stock available in the country, such as ECMO equipment, medical staffs had to directly identify and borrow equipment that they already had but did not use. According to the report of the relevant authorities, there are 9,800 mechanical ventilators and 350 ECMO equipment in the country, but there is no system in place to 
relocate them as needed in the event of a disaster in a particular region. Therefore, based on the nationwide distribution of essential equipment for critical care, measures are urgently needed to identify the areas or institutions required and to move and operate the equipment properly.

As the number of confirmed and seriously ill patients in Daegu and Gyeongsangbuk-do increased sharply, the government authorities asked the tertiary general hospital in Seoul and Gyeonggi-do, where the tertiary general hospital is the largest, to prepare and expand beds to treat critically ill patients caused by COVID-19. In response, many hospitals have set up additional negative pressure isolation rooms in ICUs and prepared to treat COVID-19 critically ill patients. However, without proper system to identify the status of available beds, an appropriate transfer system, and a control tower to manage them, it is not known how efficiently these beds were operated. In the event of an unaffordable degree of serious patients within a certain area, such as in this case in Daegu, a system for transfer patients to other regions for treatment is essential. For this purpose, a medical team dedicated to transport must be established, along with identifying the presence and location of beds capable of treating critically ill patients, and transport vehicles equipped with appropriate equipment for transport.

In this COVID-19 incident, data on the status of beds for critically ill patient beds, led by government authorities, are difficult to find. The KSCCM used its network to identify available ICU beds and the number of patients being treated according to the facility's grade, but in fact there was no opportunity for this data to be used to transfer patients. For the transport of critically ill patients by infectious diseases such as $\mathrm{CO}$ VID-19, a team of dedicated medical personnel is needed. The transport medical staffs should use the necessary equipment or administer medications in emergency situations that may occur during transport. Special ambulances equipped with a negative pressure system and a mechanical ventilator are also required. There are more than 30 vehicles designed for infectious diseases and disasters with government funds in South Korea. However, no records show how effectively these vehicles were during the outbreak of COVID-19.

If COVID-19 critically ill patients occur intensively in certain areas such as the above, a control tower is essential to establish and operate necessary medical strategies and to facilitate them, and a discussion and decision-making consultative body consisting of relevant government authorities and related private organizations is required.

\section{CONCLUSIONS}

The World Health Organization declared COVID-19 pandemic on March 11, 2020, 1 day after the collaborative medical team from the KSCCM arrived at Daegu Dongsan Hospital. As of May 26, 2020, the global COVID-19 death toll is at 343,562 and South Korea's death toll is at 269. In the condition of rapid increase in critically ill patients under the global pandemic status, it can be expected that a considerable number of patients could expire without proper critical care in ICUs. If the disastrous conditions have occurred beyond the nationwide critical care resources, it cannot be helped. However, it could be a very serious problem if, despite having sufficient critical care resources, many patients died without proper critical care due to the lack of proper systematic operation. To ensure that similar problems do not recur, policies should be prepared for sporadic outbreaks of severe cases, specific regional concentrations, and nationwide mass outbreaks in disaster situations due to various causes. It is necessary to establish a systematic strategy for the proper utilization of nationwide critical care resources, operation of essential equipment, recruitment of medical personnel.

\section{CONFLICT OF INTEREST}

No potential conflict of interest relevant to this article was reported.

\section{ORCID}

Je Hyeong Kim https://orcid.org/0000-0002-8995-7460

Suk-Kyung Hong ～https://orcid.org/0000-0001-5698-0122

Younghwan Kim https://orcid.org/0000-0003-3836-8743

Ho Geol Ryu https://orcid.org/0000-0001-8952-6049

Chi-Min Park https://orcid.org/0000-0002-8496-3546

Young Seok Lee https://orcid.org/0000-0002-0144-2033

Sung Jin Hong https://orcid.org/0000-0001-9353-2644

\section{AUTHOR CONTRIBUTIONS}

Conceptualization: JHK, SKH, SJH. Project administration: JHK, SKH, YK, HGR, CMP, YSL. Writing-original draft: JHK. Writing-review \& editing: JHK, SJH.

\section{ACKNOWLEDGMENTS}

The Volunteers for Collaboration between KSCCM and Daegu 
Dongsan Hospital for COVID-19 Critically Ill Patients: ChiMin Park, MD, Samsung Medical Center; Doim Yu, RN, Asan Medical Center; Dongsu Shin, RN, Korea University Guro Hospital; Gwang Hyeon Park, RN, Korea University Ansan Hospital; Hak Jae Lee, MD, Asan Medical Center; Ho Cheol Kim, MD, Gyeongsang National University Hospital; Ho Geol Ryu, MD, Seoul National University Hospital; Huisuk Park, RN, Seoul National University Hospital; Hye Lim Kim, RN, Korea University Ansan Hospital; Hyeanji Kim, MD; Hyunggoo Kang, MD, Hanyang University Hospital; Je Hyeong Kim, MD, Korea University Ansan Hospital; Ji Sun Son, RN, Hanyang University Hospital; Jeong Sun Lee, MD; Kyuhyouck Kyoung, MD, Ulsan University Ulsan Hospital; Kyung Deok Kim, RN, Samsung Medical Center; Min Ae Keum, MD, Ulsan University Ulsan Hospital; Min Goo Kang, MD, Samsung Medical Center; Mincheol Kim, RN, Korea University Guro Hospital; Pyoyoon Kang, MD; Sam-Woo Lee, MD; Seonhee Kwon, RN, Asan Medical Center; Seungho Jung, MD, Severance Hospital; Seungwoo Chung, MD, Hanmaeum Changwon Hospital; Soohyun Bae, MD, Ulsan University Ulsan Hospital; Suk-Kyung Hong, MD, Asan Medical Center; Sung Eon Yoo, MD; Yi Seul Lim, RN, Seoul National University Hospital; Young Rock Ha, MD, Bundang Jesaeng General Hospital; Young Seok Lee,
MD, Korea University Guro Hospital; Young Uk Choi, RN, Samsung Medical Center; Younghwan Kim, MD, National Medical Center.

\section{REFERENCES}

1. Korea Centers for Disease Control and Prevention. The updates on COVID-19 in Korea as of 15 May [Internet]. Cheongju: Korea Centers for Disease Control and Prevention; 2020 [cited 2020 May 27]. Available from: https://www.cdc.go.kr/ board/board.es?mid=a30402000000\&bid=0030

2. Zhou F, Yu T, Du R, Fan G, Liu Y, Liu Z, et al. Clinical course and risk factors for mortality of adult inpatients with COVID-19 in Wuhan, China: a retrospective cohort study. Lancet 2020;395:1054-62.

3. Hong W. "Save COVID-19 critically ill patients..." KSCCM rescue Daegu [Internet]. Seoul: Doctor's News; 2020 [cited 2020 May 15]. Available from: http://www.doctorsnews.co.kr/news/ articleView.html?idxno=133867.

4. Lee J. COVID-19 cluster in Daegu: Dongsan Hospital [Internet]. Seoul: Doctor's News; 2020 [cited 2020 May 15]. Available from: http://www.doctorsnews.co.kr/news/articleView. html?idxno=133561. 